\title{
EFFECT OF INTRAPERITONEAL AND SYSTEMIC SIROLIMUS ADMINISTRATION ON POSTOPERATIVE PERITONEAL ADHESIONS IN RATS
}

\author{
Abdullah INAL ${ }^{1}$, Serdar SAYDAM ${ }^{2}$ \\ ${ }^{1}$ Bakırcay University-Cigli Training Hospital, Department of Surgery, Izmir, TURKEY. \\ ${ }^{2}$ Dokuz Eylul University Faculty of Medicine, Department of Surgery, Izmir, TURKEY.
}

Address for Correspondence: Abdullah Inal, MD, Bakırçay University-Cigli Training Hospital, Department of Surgery, Izmir, Turkey, Email: abdullahinal@gmail.com

Received: 12.08.2021; Accepted: 24.08.2021; Available Online Date: 20.09.2021

(C)opyright 2021 by Dokuz Eylül University, Institute of Health Sciences - Available online at https://dergipark.org.tr/en/pub/jbachs

Cite this article as: Inal A, Saydam S. Effect of Intraperitoneal and Systemic Sirolimus Administration on Postoperative Peritoneal Adhesions in Rats. J Basic Clin Health Sci 2021; 3: 195- 200.

\begin{abstract}
Purpose: Low incidence of surgery-induced peritoneal adhesions in posttransplant patients is a wellknown subject. The aim of this study was to investigate the role sirolimus to prevent intra-abdominal adhesions and compare the systemic and intraperitoneal efficacy of the intervention.

Methods: A total of 21 male rats randomly constituted three groups as intraperitoneal $0.015 \mathrm{mg} / \mathrm{kg}$ sirolimus administered group, $0.1 \mathrm{mg} / \mathrm{kg}$ oral sirolimus administered group, and control group. A surgical peritoneal adhesion model was performed. All rats were euthanized on postoperative 7 . day for macroscopic adhesion evaluation and histopathologic examination.

Results: Intraperitoneal sirolimus administration significantly reduced adhesion formation. On the other hand, one-dose systemic administration did not show significant difference compared with control group. Conclusions: Intraperitoneal sirolimus administration at therapeutic doses significantly reduces postoperative intraabdominal adhesions in rat model.

Keywords: Surgery-Induced Tissue Adhesions, Sirolimus, Surgical Adhesions
\end{abstract}

\section{INTRODUCTION}

Postoperative intra-abdominal adhesion formation occurs frequently, and it may develop after any of intraperitoneal intervention. Complications which arise due to postoperative intra-abdominal adhesions include bowel obstruction, chronic abdominal and pelvic pain, infertility, adhesion related prolonged operation durations and inadvertent enterotomies, intra-operative hemorrhage, enteric fistula formation, conversion from laparoscopy to laparotomy, surgical site infection and increased hospital stay (1). There are no standard prophylactic measures for postoperative adhesion prevention. Fibrinolytics, antioxidants, anticoagulants, mechanical barriers have been proposed for adjuvant therapy for reduction of postoperative peritoneal adhesions (2). Inflammatory response seems to have prominent contribution to adhesion formation. Any kind of peritoneal insult initiates the process leads to peritoneal adhesion. There are three main pathways suggested for adhesion formation. Peritoneal injury triggers an inflammatory response along with coagulative state. Inflammation results with vascular permeability and exudation. Fibrinogen leaks to peritoneal space, interacts with fibronectin and forms fibrin gel matrix. Inflammatory cells, mainly 
macrophages and T-lymphocytes, diffuse into this matrix, secretes proinflammatory cytokines which inhibits matrix metalloproteinases and fibrinolysis. Thus, through aberrant remodeling of extracellular matrix and insufficient fibrinolysis adhesions become organized (3). Immunosuppressant agents may reduce postoperative intraperitoneal adhesions through suppression of inflammatory pathways $(4,5)$. Sirolimus is the generic name of natural byproduct rapamycin, which is produced by Streptomyces hygroscopicus. While sirolimus is a carbocyclic, lactone-lactam macrolide antibiotic isolated for antifungal properties, subsequently exhibit prominent immunosuppressive and antitumoral activities. All these immunosuppressive, antiproliferative and antifungal roles have common mechanism of action: through inactivation of mammalian target of rapamycin (mTOR) (6). Recently sirolimus has proven its efficacy in posttransplantation polydrug immunosuppression regimen with an acceptably low adverse effect profile. It has gain place in coatings of coronary and peripheral artery vascular stents to prevent stent restenosis by inhibiting intimal hyperplasia. This study aimed to investigate local and systemic effect of sirolimus on postoperative intraabdominal adhesions.

\section{MATERIAL AND METHODS}

Dokuz Eylül University Faculty of Medicine Ethics Committee on Animal Research approved the study (Protocol no:07/2011) and study is conducted in accordance with National Code for Ethics Committees on Animal Research and directives of Ethics Committee of the Dokuz Eylül University. Twenty-one male Wistar-Albino rats weighing 300 to $350 \mathrm{~g}$ were supplied by Dokuz Eylül University Animal Laboratory. At the beginning of study animals were 12 weeks old. Rats were kept at standard laboratory environment (temperature 20 to $24^{\circ} \mathrm{C}, 12 \mathrm{~h}$ light/12h dark cycle, humidity-controlled conditions with relative humidity 50 to $60 \%$ ) Animals were fed with standard rodent pellet and tap water ad libitum throughout the study. Health status of animals was not tested specifically beforehand but observed by physical examination on daily basis. A preoperative fasting for 8 hours was employed and standard diet resumed at 8 . hour postoperatively. Animals were randomly assigned to three experiment groups from randomly allocated cages. Exclusion criteria were determined as premature animal death, post-surgical complications which leads to erroneous results or inhumane outcomes, such as evisceration and deficiencies in experimental model execution.

\section{Operative Technique}

Experimental model was carried out in one session on all of animals. Anesthesia was induced by intramuscular infusion of $50 \mathrm{mg} / \mathrm{kg}$ ketamine (Ketalar ${ }^{\circledR} 50 \mathrm{mg} / \mathrm{mL}$, Eczacıbaşı, Istanbul, Turkey) along with $5 \mathrm{mg} / \mathrm{kg}$ xylazine (Rompun® $23.32 \mathrm{mg} / \mathrm{mL}$, Bayer, Istanbul, Turkey). In supine position abdomen was scrubbed with chlorhexidine solution. Abdominal mid-line was shaved and surgical skin preparation was made with $10 \%$ povidone-iodine solution. Following sterile draping abdomen was exposed through a $2.5 \mathrm{~cm}$ midline incision. All steps of surgical intervention were conducted in aseptic conditions. Anterior wall of caecum was isolated and abrasion with a gauze dissector was evidenced with punctate petechial hemorrhage. Anterior parietal peritoneum coinciding caecum was deserosalized. Animals were cleaned free of blood with chlorhexidine solution postoperatively. No animal was excluded from the study.

First Group (Controls): After adhesion model was created $1 \mathrm{~mL}$ of $0.9 \% \mathrm{NaCl}$ solution dispensed in peritoneal abrasion site, then abdomen was closed.

Second Group (Intraperitoneal sirolimus): After adhesion model was created $0.015 \mathrm{mg} / \mathrm{kg}$ sirolimus (Pfizer Inc., Philadelphia, PA) suspended in $1 \mathrm{~mL}$ $0.9 \% \mathrm{NaCl}$ solution dispensed in peritoneal abrasion site, then abdomen was closed.

Third Group (Systemic sirolimus): One hour prior to surgical intervention following general anesthesia, $0.1 \mathrm{mg} / \mathrm{kg}$ sirolimus was administered to gastrointestinal tract by an oral gavage tube.

Anterior abdominal wall incision was closed with $3 / 0$ polypropylene continuous sutures and skin was closed with $4 / 0$ polypropylene intradermal sutures. Since it has been previously confirmed by others $(7,8)$ that no further adhesions are developed after postoperative 7 days, rats were euthanized on postoperative 7. day, considering animal welfare. Adhesion formation was evaluated through autopsy.

\section{Evaluation}

On postoperative 7. day all rats were randomly allocated for surgical exploration and evaluated by a distinct surgeon who is blinded to the study with a 


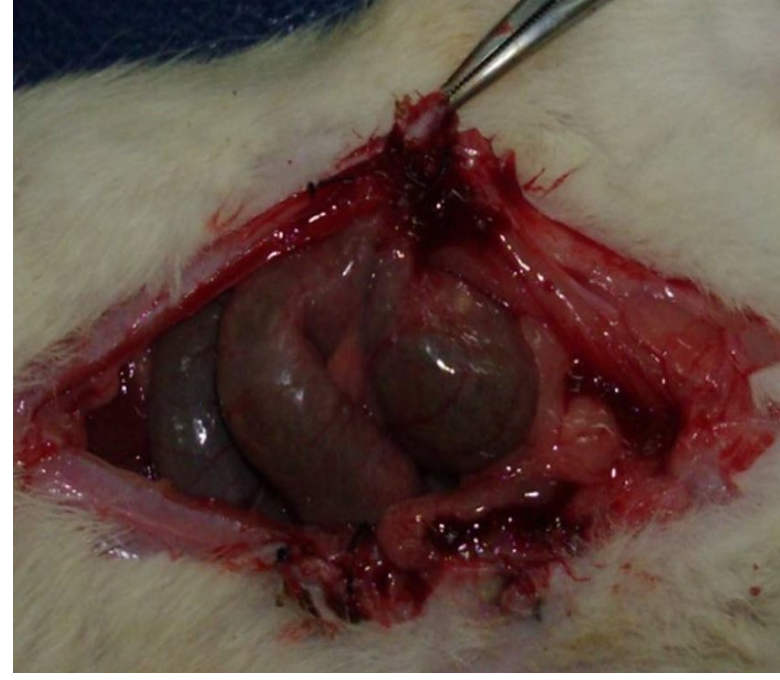

Figure 1. Dense intraabdominal adhesions and fibrous reactive tissue in a rat from control group is prominent.

macroscopic adhesion scoring system, in one session. Rats in which adhesions developed, peritoneal bands excised with affected organs, and in rats with no adhesion, ceacal anterior wall and parietal peritoneum with whole abdominal wall including all layers of except from skin excised for pathologic evaluation (Figure 1,2).

Specimens were fixed at $4^{\circ} \mathrm{C}$ in $10 \%$ formalin, afterwards embedded in paraffin and $5 \mu \mathrm{m}$ sections were prepared. Sections were stained with hematoxylin eosin then scored with light microscopy. Adhesions were evaluated macroscopically by a separate specialist who was blinded to study with a scale formerly described by Linsky et al. (9) (Table 1).

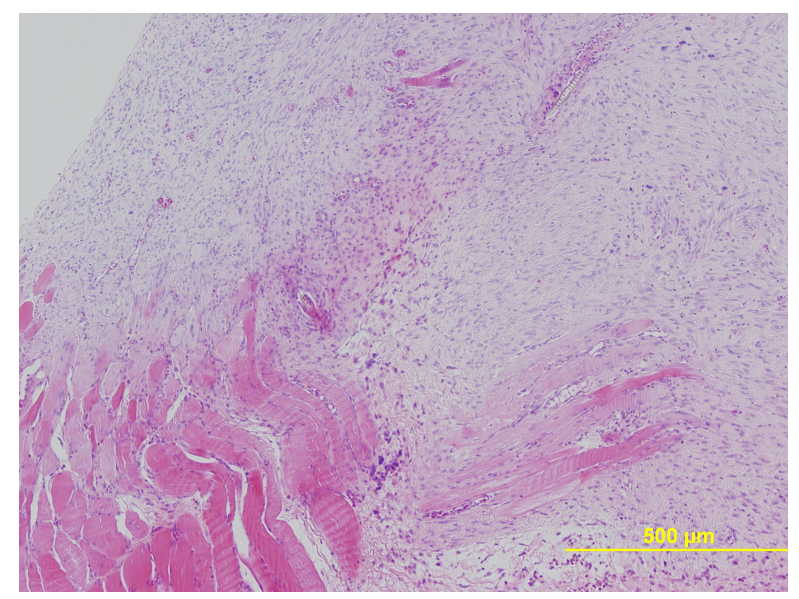

Figure 2. Microscopic view reveals inflammatory cells and fibroblasts in adhesive tissue in a sample from control group. (Heamatoxylin and Eosin stain)

\section{Statistical analysis}

Macroscopic scores are compared with KruskallWallis variance analysis with Bonferonni correction. Mann-Whitney $U$ Test was used for comparison of control group with other groups. Statistical significance level was $p<0.05$. With Bonferonni correction, $p<0.016$ was accepted significant.

\section{RESULTS}

Macroscopic evaluation scores are shown in Graphic 1. Mean intraperitoneal rapamycin group score was $0,1429(\mathrm{sd}=0,37796)$, mean oral rapamycin group score was $1,5714(\mathrm{sd}=1,61835)$ and mean control group score was $4(s d=1.82574)$. Difference in adhesion scaling scores among three groups (intraperitoneal rapamycin, oral-systemic rapamycin, control) was statistically significant $(p=0.002)$. Difference between intraperitoneal rapamycin and oral-systemic rapamycin groups was not statistically significant $(p=0.062)$. Adhesion scores of intraperitoneal rapamycin group were lower than control groups showing statistical significance $(p=0.001)$. There was no statistically significant difference between oral-systemic rapamycin and control groups $(p=0.021)$.

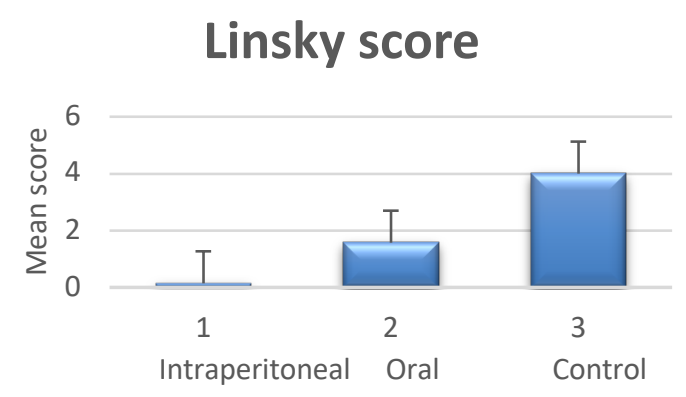

Graphic 1. Mean adhesion scores for each experiment group. Error bars are denoting standard deviation.

Although histopathologic evaluation scores were lower intraperitoneal rapamycin (mean=6,4285) and oral-systemic rapamycin groups(mean=7,7142) than control groups (mean=8.7142) there was no statistically significant difference between three groups $(p=0.246)$.

\section{DISCUSSION}

Intraperitoneal postoperative adhesions may occur virtually after any of intraabdominal intervention. In western world, with prolonged life expectation, people having increasingly more intraabdominal operations. 
Table 1. Scale to evaluate the macroscopic adhesion score by involvement, severity and resistance of adhesion.

\begin{tabular}{|l|l|l|l|l|}
\hline Linsky score & \multicolumn{3}{l|}{ Score } & \multicolumn{2}{l|}{} & \multicolumn{2}{l|}{} \\
\hline Adhesion Percentage & + & & & \\
\hline No adhesion & & + & & \\
\hline $1 \%-25 \%$ & & & + & \\
\hline $26 \%-75 \%$ & & & & + \\
\hline $76 \%-100 \%$ & & & & \\
\hline Adhesion severity & + & & & \\
\hline No adhesion & & + & & \\
\hline Filmy and avascular & & & + & \\
\hline Moderately filmy and vascular & & & & + \\
\hline Dense and significantly vascular & & & & \\
\hline Adhesion grade & + & & & \\
\hline Grade 0: no adhesion & & + & & \\
\hline Grade 1: no resistance to separation & & & + & \\
\hline Grade 2: moderate force needed for separation & & & + \\
\hline Grade 3: sharp dissection required for separation & & & \\
\hline
\end{tabular}

Morbidities of intraperitoneal adhesions such as postoperative intestinal obstruction, infertility, dyspareunia, pelvic-abdominal pain, inadvertent enterotomies, solid organ injuries, hemorrhage, bladder and ureter injuries and conversion from laparoscopy may also result in mortality. Increasing number of surgeons are getting involved with legal issues due to delays in diagnosis and treatment, and complications of secondary surgeries.

There are a number of preventive measures proposed for postoperative intraabdominal adhesions. Significant part of them are mechanic barriers. However, there are no widely accepted material because of some factors like costeffectiveness, difficulty in application, inadequate effectiveness, enteric fistula formation and anastomotic failure. Fibrinolytic agents have been shown to prevent adhesions successfully on experimental models but serious hemorrhagic complications limited its clinical use.

Crucial role of immune system in the center of pathogenesis of intraperitoneal adhesion formation proposed immunomodulation as an effective way for adhesion prevention. Inhibition of IL-1 and TNF- $\alpha$ (10), TGF- $\beta_{1}$ (11) and as an anti-inflammatory cytokine IL-10 administration $(12,13)$ significantly prevents adhesions. IL- 6 also has been shown to significantly increase the intraperitoneal adhesions $(14,15)$. Former studies with immunosuppressive agents showed adhesion prevention with tacrolimus in a bowel transplantation model (4), with sirolimus in an intraabdominal prosthetic graft model (16) and parietal synthetic mesh model (17).

Sirolimus is a new generation immunosuppressive drug with low and well-defined adverse effect profile. Sirolimus have been used for long period of time to lower the corticosteroid and cyclosporine doses and reduce their adverse effects in renal transplant patients.

The study aimed to investigate the systemic and topical effects of sirolimus on intraperitoneal adhesions. We used standard loading dose $0.1 \mathrm{mg} / \mathrm{kg}$ sirolimus as systemic administration recommended for transplantation immunosuppression, which is a quite lower dose than the dose, which is used by previous studies with sirolimus. Since the bioavailability of sirolimus is $15 \%$ and peritoneal absorption is $100 \%$, we used $0.015 \mathrm{mg} / \mathrm{kg}$ sirolimus suspended in sterile saline intraperitoneally. Thus, similar systemic doses are obtained for comparison. Adhesions are shown to be present in postoperative day 7 and persist thereafter, in previous studies (18- 
22). We achieved to show significant reduction in peritoneal adhesion by topical sirolimus administration.

Histopathologic evaluation did not reveal significant difference between three groups. This may be due to that histopathologic evaluation reflects postoperative 7. day situation but adhesion formation starts beforehand. Histologic appearance has limited value in clinical consequences.

Via its vast varied immunosuppressive, antiinflammatory, antiangiogenic, antiproliferative, antifibrotic properties, sirolimus may prevent abdominal adhesions by combination of factors. Though, impairment in wound healing is well-known effect of sirolimus $(23,24)$, we did not observe any wound healing problem or any evidence of infection in any of animals.

\section{CONCLUSION}

In conclusion, this study showed that intraperitoneal sirolimus administration significantly reduce postoperative intraperitoneal adhesions in a rat model in therapeutic dose range. This finding may offer an effective and safe option for postoperative adhesion prevention.

Conflict of interests: The authors declared no potential conflicts of interest with respect to the research, authorship, and/or publication of this article.

Ethical Aproval: Dokuz Eylül University Faculty of Medicine Ethics Committee on Animal Research approved the study (Protocol no:07/2011) and study is conducted in accordance with National Code for Ethics Committees on Animal Research and directives of Ethics Committee of the Dokuz Eylül University.

Funding: The authors received no financial support for the research, authorship, and/or publication of this article.

Author contribution: Design, fundings, materials, data collection and processing, literature review and writing of study is conducted by Abdullah Inal; Conception, supervision, analysis-interpretation and critical review of the study is conducted with Abdullah Inal and Serdar Saydam.

Peer-review: Externally peer-reviewed.

\section{REFERENCES}

1. van Goor H. Consequences and complications of peritoneal adhesions. Colorectal Dis 2007; 9(2): 2534.

2. Cheung JPY, Tsang HLH, Cheung JCJ: Adjuvant Therapy for the Reduction of Postoperative Intraabdominal Adhesion Formation. Asian Journal of Surgery 2009; 32(3): 180-186.

3. Cheong YC, Laird SM, Li TC. Peritoneal healing and adhesion formation/reformation. Human Reproduction Update 2001; 7(6): 556-566.
4. Wasserberg N, Nunoo-Mensah JW, Ruiz P. The Effect of Immunosuppression on Peritoneal Adhesions Formation After Small Bowel Transplantation in Rats. Journal of Surgical Research 2007; 141: 294-298.

5. Peker K, Inal A, Sayar I, et al. Prevention of intraabdominal adhesions by local and systemic administration of immunosuppressive drugs. Iran Red Crescent Med J 2013; 15(12): e14148.

6. Trepanier DJ, Gallant H, Legatt DF. Rapamycin: distribution, pharmacokinetics and therapeutic range investigations: an update. Clinical Biochemistry 1998; 31(5): 345-351.

7. W B Gaertner 1, M E Bonsack, J P Delaney Visceral adhesions to hernia prostheses. Hernia 2010; 14(4): 375-81.

8. Baptista ML, Bonsack ME, Felemovicius I, Delaney JP Abdominal adhesions to prosthetic mesh evaluated by laparoscopy and electron microscopy. J Am Coll Surg 2000; 190:271-280

9. Linsky $C B$, Diamond MP, Cunningham T, B Constantine, DeCherney AH, diZerega GS. Adhesion reduction in a rabbit uterine horn model using an absorbable barrier, TC-7. J Reprod Med 1987; 32: 17-20.

10. Kaidi A, Nazal M, Gurmchumelidze T, Ali MA, Dawe EJ, Silva YJ. Preoperative administration of antibodies against tumour necrosis factor-alpha (TNF-alpha) and interleukin-1 (IL-1) and their impact on peritoneal adhesion formation. Am Surg 1995; 61: 569-572.

11. Lucas $P$, Warecka $D$, Young $H$ Lee BY. Formation of abdominal adhesions is inhibited by antibodies to transforming growth factor-beta1. J Surg Res 1996; 65: 135-138.

12. Holschneider C, Cristoforoni $P$, Ghosh K, Punyasavatsut M, Abed E, Montz FJ. Endogenous versus exogenous $\mathrm{IL}-10$ in post-operative intraperitoneal adhesion formation in a murine model. J Surg Res 1997; 70: 138-143.

13. Montz F, Holschneider C, Bozuk M, Gotlieb WH, Martinez-Maza O. Interleukin 10: ability to minimize postoperative intraperitoneal adhesion formation in a murine model. Fertil Steril 1994; 61: 1136-1140.

14. Saba A, Kaida A, Godziachvili V, Dombi GW, Dawe EJ, Libcke JH, Silva YJ. Effects of IL-6 and its neutralising antibodies on peritoneal adhesions formation and wound healing. Am Surg 1996; 62: 569-772.

15. Buyalos RP, Funari VA, Azziz R, Watson JM, Martinez-Maza O. Elevated interleukin-6 levels in 
peritoneal fluid of patients with pelvic pathology. Fertil Steril 1992; 58: 302-306.

16. Kanko M, Ozbudak E, Ozerdem A. Effect of Sirolimus in the Prevention of Adhesions Around Intraabdominal Prosthetic Graft. World J Surg 2006; 30: 1648-1652

17. Maciver $\mathrm{AH}, \mathrm{McCall} \mathrm{MD}$, Edgar RL, et al. Sirolimus drug-eluting, hydrogel-impregnated polypropylene mesh reduces intra-abdominal adhesion formation in a mouse model. Surgery 2011; 150(5): 907-15.

18. Yelian FD, Shavell VI, Diamond MP. Early demonstration of postoperative adhesions a rodent model. Fertil Steril 2010; 93(8): 2734-2737.

19. L. Holmdahl, B. Risberg, D.E. Beck, et al. Adhesions: pathogenesis and prevention-panel discussion and summary. Eur J Surg Suppl 1997; 557: 56-62.

20. E.S. Harris, R.F. Morgan, G.T. Rodeheaver. Analysis of the kinetics of peritoneal adhesion formation in the rat and evaluation of potential antiadhesive agents. Surgery 1995; 117: 663-669.

21. R.C. Dunn, M. Mohler. Effect of varying days of tissue plasminogen activator therapy on the prevention of postsurgical adhesions in a rabbit model. J Surg Res 1993; 54: 242-245

22. H. Orita, M. Fukasawa, W. Girgis, G.S. diZerega. Inhibition of postsurgical adhesions in a standardized rabbit model: intraperitoneal treatment with tissue plasminogen activator. Int J Fertil 1991; 36: 172-177 23. Willems MCM, van der Vliet JA, de Man BM, van der Laak JAWM, Romme RMLM, Hendriks T. Persistent effects of everolimus on strength of experimental wounds in intestine and fascia. Wound Rep Reg 2010; 18: 98-104

24. Ekici $Y$, Emiroglu R, Ozdemir $H$, Karakayali $H$, Haberal M. Effect of rapamycine on wound healing: an experimental study. Transplant Proc 2007; 39: 1201-3. 